\title{
Efectos de las actividades en la naturaleza en la predicción de la satisfacción de la Educación Física Effects of outdoor activities in predicting Physical Education satisfaction
}

\author{
Antonio Baena Extremera*,Antonio Granero Gallegos** \\ *Universidad de Murcia (España), **Consejería de Educación. Junta de Andalucía (España)
}

Resumen. El objetivo de este trabajo ha sido conocer cómo pueden influir las actividades en el medio natural en la predicción de la satisfacción hacia la Educación Física. La muestra fue de 125 alumnos de $4^{\circ}$ de la ESO de Educación Secundaria divididos en un grupo experimental ( $n=76$ ) y grupo control (n=49). Se utilizó un cuestionario compuesto por la Basic Psychological Needs in Exercise Scale, el factor de Motivación Intrínseca del Sport Motivation Scale, y Sport Satisfaction Instrument. Se realizó un programa de intervención con Educación de Aventura con 18 sesiones distribuidas en tres unidades didácticas y se tomaron medidas antes (pretest) y después de la intervención (postest). Se realizaron análisis descriptivos y modelos de ecuaciones estructurales. Tras el Programa de Aventura mejoran todas las variables excepto el aburrimiento, sobre todo la relación con los demás. La autonomía predice principalmente la motivación intrínseca, y ésta la satisfacción/diversión en el alumnado.

Palabras clave. Educación de aventura, necesidades psicológicas, motivación, satisfacción, educación.

\begin{abstract}
The aim of this study was to determine the effect of outdoor activities in the prediction of Physical Education satisfaction. The sample comprised 125 students of high-school students assigned into an experimental ( $\mathrm{n}=76)$ and a control group ( $n=49)$. Participants completed the Basic Psychological Needs in Exercise Scale, the Intrinsic Motivation factor of the Sport Motivation Scale, and the Sport Satisfaction Instrument. The intervention program consisted on 18 Adventure Education sessions divided on three teaching units. Before (pre-test) and after (post-test) intervention measurements were obtained. Descriptive analyzes and Structural Equation Modeling were used to analyze the data. Following the Adventure Program, all variables improved but boredom, especially the relationship with others. Autonomy predicts mainly intrinsic motivation, which in turn, predicts satisfaction/enjoyment in students.
\end{abstract}

Keywords. Adventure education, psychological needs, motivation, satisfaction, education.

\section{Introducción}

Autores como Flintoff y Scraton (2001), MacPhail, Kirk, y Eley (2003), Mowling, Brock, Eiler, y Rudisill (2004) y Rikard y Banville (2006), concluyeron en sus trabajos que el alumnado, en general, encuentra las sesiones de Educación Física (EF) aburridas y monótonas, pues prima en ellas la repetición de actividades año tras año. A esto habría que sumarle además la presencia de un profesorado de EF desfasado y desvinculado de los intereses de los jóvenes de hoy, quienes solicitan más variedad de actividades deportivas, un mayor nivel de desafío en las actividades de EF, así como diversas actividades de aventura al aire libre.

Desde que la Ley Orgánica General del Sistema Educativo de 1990 incluyó en el currículum de EF las actividades en el medio natural (AMN) en España, el interés que estos contenidos han despertado entre el profesorado y el alumnado ha aumentado notablemente hasta nuestros días. Los motivos de este acrecentamiento son diversos, pero uno de los más importantes son los beneficios educativos que atesoran y que, sin duda, ya han sido objeto de estudio en diversas investigaciones (Caballero, 2012; Granero \& Baena, 2011; Moos \& Honkomp, 2011); y, por otro lado, la novedad e interés que despiertan para el alumnado, acercándose a los intereses y motivaciones de los estudiantes.

Una de las claves para conseguir estudiantes con interés hacia el aprendizaje es tratar que se encuentren satisfechos con la enseñanza que reciben (Baena-Extremera, Granero-Gallegos, Bracho-Amador, \& PérezQuero, 2012). Ntounamis (2005) concluyó que cuando un alumno se encuentra satisfecho y se divierte en clase tiende a estar intrínsecamente motivado, lo que supone una mayor participación en EF e, incluso, una mayor práctica de actividad física en su tiempo libre. Por tanto, es importante tener en clase estudiantes que se sienten satisfechos con sus clases y que además, se encuentran motivados.

La motivación, se entiende como un modulador que puede afectar a otras variables que influyen en el aprendizaje. Siguiendo la teoría de la autodeterminación (Deci \& Ryan, 1985), las personas presentan tres necesidades psicológicas básicas: autono-mía, competencia y relación con los demás. Si éstas necesidades son sa-tisfechas a través del contexto social, puede aumentar la motivación y ésta puede presentar consecuencias positivas en el rendimiento escolar y en el bienestar y desarro-

Fecha recepción: 03-02-15- Fecha envío revisores: 03-02-15- Fecha de aceptación: 11-03-15 Antonio Baena Extremera abaenaextrem@um.es llo personal de los alumnos (Standage \& Treasure, 2002). Deci y Ryan (2000) indican además que las tres necesidades básicas, y la percepción de las mismas, podrán influir en la motivación creando una mayor motivación intrín-seca y una menor desmotivación.

Algunos trabajos han comprobado que los climas de aprendizaje en los que el docente potencia la autonomía del discente ayuda a mejorar la motivación de estos últimos (How, Whipp, Dimmock, \& Jackson, 2013; Matos, 2009). Por ello, la autonomía supone una variable importante y a tener en cuenta en la enseñanza, sobre todo cuando las leyes educativas españolas incluyen la autonomía e iniciativa personal entre las competencias básicas que el alumnado debe adquirir en la educación secundaria. Esto, además, adquiere una importancia mayor si los contenidos que se trabajan en clase, y no sólo la metodología del profesor, propician la proliferación de la autonomía en el alumno, como son el caso de las AMN.

En algunos trabajos pioneros en este ámbito (Baena-Extremera \& Granero-Gallegos, 2013; Baena-Extremera, Granero-Gallegos, \& OrtizCamacho, 2012), los investigadores pudieron comprobar cómo los alumnos, tras «disfrutar» de un programa de educación de aventura(EA) con AMN en EF, mejoraron en muchos aspectos, entre ellos la competencia, la motivación, la satisfacción, disminuyendo el aburrimiento.

En España además, apenas existen trabajos de investigación y menos aún de intervención sobre AMN, donde se puedan comprobar de forma empírica los beneficios que estos contenidos tienen en el estudiante. Por todo esto, el objetivo de este trabajo es conocer cómo pueden influir las AMN en la predicción de la satisfacción hacia la EF, a través del análisis de las necesidades psicológicas básicas del alumno y la motivación intrínseca. Tras aplicar un programa de EA, se establecen

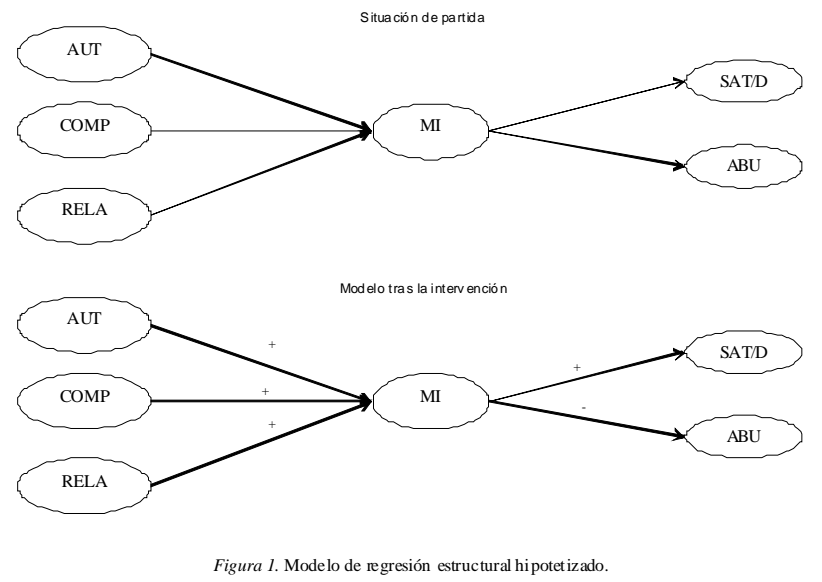


las siguientes hipótesis: 1) Se espera que se produzcan mejoras (puntuaciones medias más altas) en las variables estudiadas; 2) Se cree que se producirán cambios en la predicción de la motivación intrínseca y de la satisfacción/diversión, con incrementos positivos en estas relaciones predictivas, fundamentalmente a partir de alguna de las necesidades psicológicas básicas. El modelo hipotetizado sería el siguiente:

\section{Método}

\section{Participantes}

En esta investigación participaron un total de 125 alumnos de $4^{\circ}$ curso de Educación Secundaria Obligatoria (ESO) de España, con edades comprendidas entre 15 y 16 años $(M=15.67 ; D T=.71)$. Entre el alumnado, 59 eran chicos, siendo dos de ellos de origen inglés, uno de origen pakistaní y tres de origen boliviano; y 66 eran chicas, siendo sólo cuatro de ellas de origen inglés, pertenecientes a un centro de enseñanza pública de la provincia deAlmería (España). Las clases eran mixtas y los contenidos impartidos en el grupo experimental correspondían a un programa de EA. Los contenidos recibidos por el grupo control, durante el mismo periodo e igual número de sesiones, fueron de juegos y deportes, realizadas dentro de las instalaciones deportivas del centro.

\section{Instrumentos}

Escala de las Necesidades Psicológicas Básicas en el Ejercicio (BPNES). Se utilizó la versión validada al español y adaptada a la EF (Moreno, et al., 2008b) de la original Basic Psychological Needs in Exercise Scale (Vlacho-poulos \& Michailidou, 2006). El inventario consta de 12 ítems agrupados en tres dimensiones (cuatro ítems por dimensión): autonomía (AUT), competencia (COMP) y relación con los demás (RELA). Las respuestas fueron recogidas con una escala de ítems politómicos que oscilaba entre uno (totalmente en desacuerdo) y cinco (totalmente de acuerdo). La consistencia interna hallada en el pretest y postest resultó satisfactoria: autonomía, alfa de Cronbach (á) $=.71$ y á $=.73$; competencia á $=.73$ y á $=.73$; relación con los demás, á $=.85$ y á $=.83$

Motivación Intrínseca. Se empleó el factor de motivación intrínseca de la Escala de Motivación en el Deporte(SMS), compuesto por un total de 12 ítems. Se utilizó la versión española adaptada a la EF y (Granero-Gallegos \& Baena-Extremera, 2013; Granero-Gallegos, BaenaExtremera, Gómez-López, Sánchez-Fuentes, \& Abraldes, 2014). La escala original se denominó Échelle de Motivationdans les Sports (ÉMS; Brière, Vallerand, Blais, \& Pelletier, 1995) y fue traducida al inglés por Pelletier et al. (1995) pasando a denominarse Sport Motivation Scale (SMS). Consta de 28 ítems que medían los diferentes tipos de motivación establecidos por la teoría de la autodeterminación (Deci \& Ryan, 1985) que sugiere la explicación multidimensional de la motivación: desmotivación, motivación extrínseca (ME) y motivación intrínseca (MI). Se utilizó el factor motivación intrínseca de la versión de tres factores de esta escala. Las respuestas fueron recogidas en una escala de ítems politómicos que oscilaba entre uno (totalmente en desacuerdo) y siete (totalmente de acuerdo). La consistencia interna hallada en el pretest y postest resultó satisfactoria: motivación intrínseca, á = .92 y á = .93. La consistencia interna hallada en el presente estudio fue: motivación intrínseca, á $=.93$.

Sport Satisfaction Instrument (SSI). Se utilizó la versión española del Sport adaptada a EF (SSI-EF) (Baena-Extremera, et al., 2012) del original Sport Satisfaction Instrument (Duda \& Nicholls, 1992). El SSIEF consta de ocho ítems para medir la satisfacción intrínseca en una actividad deportiva mediante dos subescalas que miden satisfacción/ diversión (SAT/D) (cinco ítems) y aburrimiento (ABU) (tres ítems) en la práctica deportiva. En las instrucciones se pide a los sujetos que indiquen su grado de acuerdo con los ítems que reflejan criterios de diversión o aburrimiento, recogiéndose las respuestas en una escala de ítems politómicos de cinco puntos que oscila desde muy en desacuerdo (1) a muy de acuerdo (5). La consistencia interna hallada en el pretest y postest resultó satisfactoria: satisfacción/diversión, á = .94 y á = .93; aburrimiento, á $=.81$ y á $=.82$.

\section{Diseño}

Su utilizó un diseño cuasi-experimental, descriptivo y seccional. Se trabajó con grupo control no equivalente, pues la composición de los grupos de clase ya estaban establecidos por el centro educativo. Participaron cinco grupos de $4^{\circ}$ de ESO. Se realizó un sorteo para elegir al azar los tres grupos que serían experimentales, resultado ser designados los grupos A, C y E, compuestos por 24, 27 y 25 alumnos, respectivamente, formando un total de 76 personas. Los grupos designados como B y D constituyeron los dos grupos control y estaban compuestos por 26 y 23 alumnos, respectivamente, formando un total de 49 personas. Cada grupo recibió las sesiones de EF y EA por el mismo profesor durante el periodo de la investigación. Los grupos experimentales recibieron tres unidades didácticas de EA y los grupos control continuaron con el desarrollo habitual de la programación anual de la asignatura y en el mismo periodo se desarrollaron dos unidades didácticas de deportes colectivos y otra de deportes individuales, planificadas en las mismas sesiones que el programa de intervención. Para poder desarrollar el programa de EA dentro de la planificación anual del centro, se estudiaron los documentos de organización curricular de los grupos A, C y E, para evitar las perdidas de otras unidades didácticas debido a la introducción del programa de EA. Una vez estudiadas las posibilidades de modificación curricular, se solicitó permiso a los órganos competentes, siendo oficialmente autorizada por el Consejo Escolar del instituto, padres y tutores legales.

Durante las clases, se realizaron varias medidas de los factores autonomía, competencia, relación con los demás, motivación intrínseca, satisfacción/diversión y aburrimiento, utilizando los cuestionarios en papel descritos en el apartado de instrumentos: un pretest en todos los grupos antes de comenzar la intervención y un postest justo al terminar el programa de EA y las unidades didácticas del bloque de contenidos de juegos y deportes. Fueron descartados los cuestionarios de aquellos que no asistieron al menos a catorce sesiones durante el periodo en cuestión. Esto supuso la pérdida en la medida postest de 8 sujetos en el grupo experimental y 5 en el grupo control.

Para garantizar la validez interna, externa y ecológica de este diseño, se revisó cuidadosamente la mortalidad experimental y la selección de los grupos control y experimental. Además, se evitó el posible efecto del pretest y del experimentador, como se puede apreciar en el siguiente apartado. Asimismo, se aseguró que los grupos estudiados hubieran tenido experiencias de actividades en la naturaleza en cursos anteriores (de ahí que se utilizaran alumnos de $4^{\circ}$ curso), para evitar el efecto de la novedad del programa.

El programa se ubicó dentro del bloque de contenidos de AMN en Educación Secundaria Obligatoria. Para desarrollar el programa de EAse siguieron las características descritas por Hattie, Marsh, Neill, y Richards (1997), situándonos en la propuesta de Rhonke (1989) para el diseño de las sesiones. El centro educativo elegido contaba con la infraestructura mínima para poder desarrollar contenidos como trepa, escalada, tirolina, rapel, etc. El programa se llevó a cabo por el profesor de EF, asesorado por un profesor experto en metodología de enseñanza y EA, encargado del diseño el programa de intervención, tras diversas evaluaciones iniciales. Las tareas se diseñaron para que todos los discentes tuvieran posibilidad de alcanzar el éxito, pues de esta manera, se incrementarían los efectos positivos del programa.

\section{Análisis estadístico}

Se llevó a cabo un análisis descriptivo y de correlación entre todas las variables. Los análisis de ítems, homogeneidad y estructura interna, correlación (coeficiente de Pearson) y consistencia interna (alfa de Cronbach) de la escala. Se han calculado los índices de asimetría y curtosis siendo, en general, próximos a cero y $<2$. A continuación se llevó a cabo un análisis de las puntuaciones de diferencia o análisis de puntuación de ganancia entre el pretest y el postest para cada alumno, analizando a continuación las diferencias en un sentido único de tratamiento utilizando la prueba T de Student para muestras relacionadas. En los diferentes análisis se ha tenido en cuenta el valor de la prueba de Levene, de homogeneidad de las varianzas. Para todo ello se empleó el 
SPSS versión 17.0. Finalmente se realizó un análisis de ecuaciones estructurales con las puntaciones del grupo experimental con el programa LISREL 8.80.

\section{Resultados}

\section{Análisis preliminar}

Con objeto de comprobar si se partía de dos grupos homogéneos, se llevó a cabo un estudio preliminar en el que se analizaron las diferencias inter-grupos mediante la Prueba $T$ para muestras independientes. En la Tabla 1 se pueden comprobar los datos más relevantes y que hacen referencia a los descriptivos de las medidas pretest, así como la prueba $T$, que demuestra que no existen diferencias significativas en ninguna de las dimensiones entre el grupo experimental y el grupo control. Se trata, por tanto, de dos grupos homogéneos.

Con las medidas pretest y postest del grupo experimental se realizó un análisis de correlaciones bivariadas (Tabla 2) para comprobar las relaciones existentes entre los diferentes constructos. La motivación intrínseca presentó puntuaciones medias altas sobre una escala de siete puntos, sobre todo en el postest. En la escala de satisfacción, los valores más altos correspondieron a la satisfacción/diversión y más bajos en el aburrimiento; en este caso, en el postest la satisfacción/diversión puntuó más alto y el aburrimiento más bajo que en el prestest. Respecto a las necesidades psicológicas básicas, las tres escalas puntuaron más alto en el postest, correspondiendo las puntuaciones medias superiores a la relación con los demás, seguidas de la competencia y de la autonomía. En la Tabla 2 también se exponen los datos de correlación entre las diferentes subescalas estudiadas, tanto del pretests como del postest. Resalta la alta y positiva correlación entre la motivación intrínseca y la satisfacción/diversión, autonomía y competencia. La satisfacción/diversión también presentó altas correlaciones con la autonomía y la competencia. Asimismo, la competencia correlacionó positiva y significativamente con la autonomía y con la relación con los demás. Entre las relaciones negativas resaltan las del aburrimiento con la satisfacción/diversión y la motivación intrínseca.

Tabla 1

Media (M) y desviación típica (DT) de la medida pretest. Prueba Tde Student para muestras independientes según grupo experimental o grupo control

\begin{tabular}{lcccccc}
\hline \multirow{2}{*}{ Subescalas } & \multicolumn{2}{c}{ Grupo experimental } & \multicolumn{2}{c}{ G rupo control } & \multicolumn{2}{c}{$\begin{array}{c}\text { Prueba } \boldsymbol{T} \text { para la } \\
\text { igual dad de medias }\end{array}$} \\
\cline { 2 - 7 } & $M$ & $D T$ & $M$ & $D T$ & $t$ & $\begin{array}{c}\text { Signific. } \\
\text { (bilateral) }\end{array}$ \\
\hline Motivación intrínseca & 4.65 & 1.23 & 4.58 & 1.22 & 3.98 & .112 \\
Satisfacción/diversión & 3.57 & .92 & 3.63 & .90 & 4.65 & .645 \\
Aburrimiento & 2.36 & 1.10 & 2.40 & 1.12 & 5.38 & .201 \\
Aut onomía & 3.02 & .93 & 2.95 & .99 & 3.63 & .121 \\
Competencia & 3.31 & .92 & 3.30 & .92 & 3.21 & .201 \\
Rel ación con los demás & 3.57 & .95 & 3.49 & .97 & 5.28 & .743 \\
\hline
\end{tabular}

Correlación de las subescalas anal izadas, pretest y postest del grupo experimental

\begin{tabular}{|c|c|c|c|c|c|c|}
\hline Subescal as & 1 & 2 & 3 & 4 & 5 & 6 \\
\hline 1. Motivación intrínseca & & $.61^{* *}$ & $-.37 * *$ & $.56^{* *}$ & $.49 * *$ & $.32 * *$ \\
\hline 2. Satisfacción/divers ión & $.64 * *$ & & $-.48 * *$ & $.56^{* *}$ & $.53 * *$ & $.39 * *$ \\
\hline 3. Aburrimiento & $-33^{* *}$ & $-.51^{* *}$ & & $-.24 * *$ & $-.22^{* *}$ & $-.21^{* *}$ \\
\hline $\begin{array}{l}\text { 4. Autonomía } \\
\text { 5. Competencia }\end{array}$ & $\begin{array}{l}.61^{* *} \\
.56 * *\end{array}$ & $\begin{array}{l}.56 * * \\
.55^{* *}\end{array}$ & $\begin{array}{l}-.22 * * \\
-.20^{* *}\end{array}$ & $.67 * *$ & $.62 * *$ & $\begin{array}{l}.50^{* *} \\
.55^{* *}\end{array}$ \\
\hline 6. Rel ación con los demás & $.45 * *$ & $.40^{* *}$ & $-.17 * *$ & $.51^{* *}$ & $.58 * *$ & \\
\hline
\end{tabular}

6. Relación con los demás

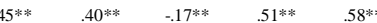

* * La correlación es significativa al nivel 01. Diagonal superior pretest, diagonal inferior postest. Pre pretest; post $=$ postest.

\section{Efectos de la intervención}

Al comparar los resultados obtenidos por el grupo experimental y el grupo control en el pretest y en el postest la prueba de $T$ arroja diferencias significativas entre las medias de las nueve dimensiones estudiadas en el grupo experimental (Tabla 3). En el grupo control no se hallaron diferencias significativas en ninguna de las subescalas. La puntuación de ganancia se calculó restando la puntuación media del postest a la puntuación media del pretest, tanto en el grupo experimental, como en el grupo control, respectivamente: postest-pretest=puntuación de ganancia. Las máximas diferencias se hallaron en la relación con los demás $\left(t_{147}=15.72 ; p<.001\right)$, la satisfacción/diversión $\left(t_{147}=14.65\right.$; $p<.001)$, la motivación intrínseca $\left(t_{148}=12.14 ; p<.000\right)$, con puntuaciones de ganancia de $.67, .60$ y .56. Le siguen la competencia $\left(t_{148}=11.32\right.$; $p<.001$ ) y la autonomía ( $\left.t_{147}=6.56 ; p=004\right)$, con una ganancia de .51 y
.36 entre los participantes del grupo experimental, respectivamente. En la dimensión de aburrimiento se presenta una importante puntuación de ganancia negativa (-.47), pues la medida tras el programa de aventura fue menor al pretest, con diferencias significativas $\left(t_{147}=-9.46 ; p=.002\right)$.

Respecto a la hipótesis de estudio, se puede decir que el programa ha resultado eficaz, pues el grado de cambio en todas las subescalas estudiadas es significativo.

\begin{tabular}{|c|c|c|c|c|c|c|c|c|}
\hline \multirow[b]{2}{*}{ Dimensiones } & \multicolumn{2}{|c|}{$\begin{array}{c}\text { Grupo } \\
\text { Experimental }\end{array}$} & \multicolumn{2}{|c|}{$\begin{array}{l}\begin{array}{c}\text { Grupo } \\
\text { control }\end{array} \\
\end{array}$} & \multirow{2}{*}{$\begin{array}{c}\begin{array}{c}\text { Grupo } \\
\text { Experimental }\end{array} \\
\text { Puntuación } \\
\text { de ganancia }\end{array}$} & \multirow{2}{*}{$\begin{array}{c}\begin{array}{c}\text { Grupo } \\
\text { control }\end{array} \\
\text { Puntuación } \\
\text { de ganancia }\end{array}$} & \multicolumn{2}{|c|}{$\begin{array}{l}\text { Prueba T para la } \\
\text { igualdad de medias }\end{array}$} \\
\hline & $M$ & $D T$ & M & $D T$ & & & $t$ & $\begin{array}{c}\text { Signific. } \\
\text { (bi lateral } \\
\text { ) }\end{array}$ \\
\hline Motivación intrínseca & 5.21 & 1.33 & 455 & 1.18 & .56 & -.03 & 12.14 & .000 \\
\hline Satisfacción/diversión & 4.17 & .90 & 3.65 & .94 & 60 & .02 & 14.65 & .000 \\
\hline Aburrimiento & 1.89 & 1.01 & 245 & 1.07 & -.47 & .05 & -9.46 & .002 \\
\hline Autonomía & 3.38 & .94 & 292 & .93 & .36 & -.03 & 6.59 & .004 \\
\hline Competencia & 3.82 & .88 & 325 & .94 & .51 & -.05 & 11.32 & . \\
\hline Relación con los demás & 4.24 & .89 & 353 & .98 & .67 & .04 & 15.72 & .000 \\
\hline
\end{tabular}

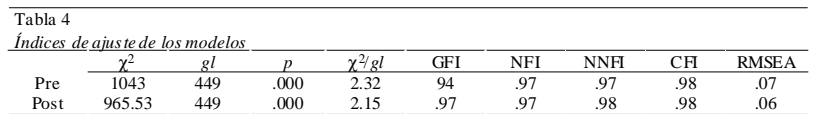

Modelo de Ecuaciones Estructurales con el Grupo Experiemental

Para comprobar la relación predictiva entre las variables analizadas se pasó a testar la validez de constructo del modelo establecido. Debido a la falta de normalidad de los datos, este análisis se llevó a cabo utilizando el método de estimación weighted least squares (WLS) para variables ordinales del programa LISREL 8.80 (Jöreskog \& Sörbom, 2003). La matriz de correlaciones policóricas y la matriz de covarianzas asintóticas fueron utilizadas como input para el análisis de los datos. Se hipotetizó para cada escala un modelo de medida consistente en un modelo de factores que asumió la existencia de las variable latentes según los instrumentos originales descritos en el apartado correspondiente.

El ajuste fue evaluado con una combinación de índices de ajuste absolutos y relativos. Entre los absolutos, se utilizó el valor $p$ asociado con el estadístico chi cuadrado $\left(\chi^{2}\right)$ y la ratio entre $\chi^{2}$ y grados de libertad $(g l)\left(\chi^{2} / g l\right)$. Asimismo, se ha calculado el GFI (índice de bondad de ajuste), cuyo valor debe ser igual o superior a .90 para considerar mínimamente aceptable el ajuste de un modelo, aunque autores como Hooper, Coughlan y Mullen (2008) consideran valores >.95 para un mejor ajuste. Entre los índices relativos se ha utilizado el NFI (índice de ajuste normalizado), el NNFI (índice de ajuste no normativo) y CFI (índice de ajuste comparativo). En los índices incrementales se considera que valores $>.95$ indican un buen ajuste (Hu \& Bentler, 1999). Autores como Kline (2005) recomiendan la utilización de RMSEA(error de aproximación cuadrático medio) y, según Hu y Bentler (1999) un valor d».06 indicaría un buen ajuste, aunque Steiger (2007) apunta que $<.07$ es un valor límite de consenso. Los parámetros estimados se consideran significativos cuando el valor asociado al valor $t$ es superior a 1.96 $(p<.05)$.

Con el objeto de analizar las relaciones e interacciones existentes entre las variables pertenecientes al modelo que se plantea, se ha utilizado el Modelo de Ecuaciones Estructurales. Atendiendo a las recomendaciones de autores como Markland (2007) o Levy y Hancock (2007), de formular y analizar varios modelos si los datos así lo recomiendan y de reportar los resultados más relevantes, se llevaron a cabo diversos análisis de modelos. Teniendo en cuenta la hipótesis de partida (2), la cual se ajusta a los marcos teóricos existentes, se buscó la predicción de las necesidades psicológicas básicas sobre la motivación intrínseca y ésta, sobre la satisfacción/diversión y el aburrimiento. De esta forma, se obtuvo como resultado final un modelo con buen ajuste según las variables estudiadas, tal como se representa en la Figura 1. En relación a esta ecuación, se llevó a cabo un análisis antes (pretest) y después (postest) de llevar a cabo el trabajo de intervención, obteniéndose los valores reflejados en la Tabla 4.

Los datos aquí reflejados se ajustan a los parámetros establecidos, por lo que se pueden aceptar como buenos los modelos propuestos 


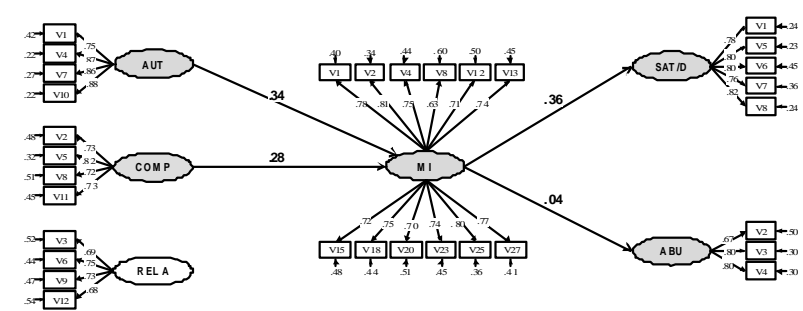

Figura 1. Pretest (grupo-experimental) de anál isis confirmat orio compuesto por seis factores hi potet izados. Los circulos representan los constructos latentes y los cuadrados representan las variables medidas. Todos los parámetros son estandarizados y significativos en $p<.05$. AUT: autonomí; COMP: competencia;

RELA: relación con los demás; MI: motivación intŕńneca; SAT/D: satisfacción /diversión; ABU: aburrimiento.

(Hu \& Bentler, 1999), tanto antes como después del programa de intervención. Los datos ofrecidos en las Figuras 1 y 2 muestran seis variables latentes con un total de 32 variables observadas.

En la Figura 1 se muestran los resultados del modelo pretest y se observa como la autonomía (.34) y la competencia (.28), predicen positivamente la motivación intrínseca, mientras que la relación con los demás queda fuera del modelo para que los ajusten sean adecuados. A partir de ahí, la motivación intrínseca se muestra buena predictora de la satisfacción/diversión (.36) y la relación respecto al aburrimiento apenas es reseñable (.04).

En la Figura 2 se exponen los resultados del modelo POSTESTy se comprueba que la relación con los demás se incluye en el modelo, alcanzando ahora un ajuste adecuado. Los tres factores de la escala de necesidades psicológicas básicas se muestran predictoras de la motivación intrínseca, sobre todo la autonomía (.71) y la competencia (.68), aunque la relación con los demás alcanza un valor predictivo de.54. Tras el desarrollo del programa de intervención la motivación intrínseca predice de una manera importante la satisfacción/diversión (.72) en clase de EF, mientras que ahora si es destacable la relación de predicción negativa respecto al aburrimiento (-.46)

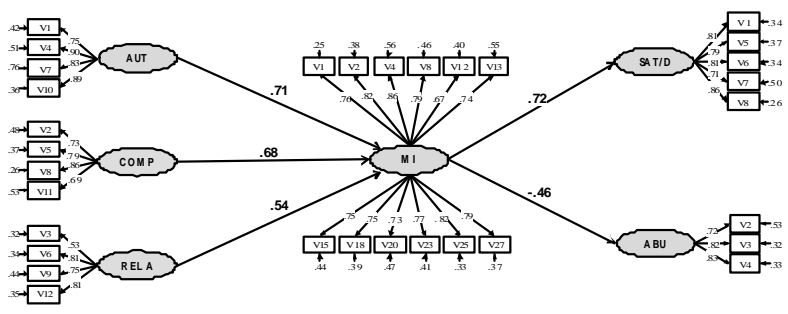

Figura 2. Postest (grupo-experimental) de análisis confirmatorio compuesto por seis factores hipotetizad os. Los circulos representan los constructos latentes y los cuadrados representan las variables medidas. Todos los parámetros son estandarizados y significativos en $p<.05$. AUT: autono mía; COMP: competencia; RELA
relación con los demás; MI: motivación intrínseca; SAT/D: satisfacción /divers ión; ABU: aburimiento

\section{Discusión}

El objetivo de este trabajo ha sido conocer cómo pueden influir las AMN en la predicción de la satisfacción/diversión en EF. Se tiene conocimiento que estos contenidos tiene efectos positivos diversos en el alumnado (Gatzemann, Schweizer, \& Hummel, 2008; Granero \& Baena, 2007; Wurdinger \& Steffen, 2003), y en el presente trabajo se demuestran empíricamente estos beneficios sobre las variables estudiadas.

Como apuntan Flintoff y Scraton (2001), el alumnado de secundaria echa en falta, para una mayor motivación, actividades de desafío y reto y que aporten nuevas experiencias. Tras el trabajo de intervención con el programa de EA, se ha podido comprobar una mejora importante en la relación con los demás, la satisfacción, la motivación intrínseca, seguido de la competencia y algo menos en la autonomía.

La autonomía es de las necesidades psicológicas básicas que menos mejora tras recibir este programa de EA; no obstante, la mejora es importante. El aumento de la puntuación de ganancia es un dato alentador, ya que la motivación de un alumno en EF dependerá de diversos factores, entre los que destaca principalmente la autonomía. A nivel educativo, y enlazando con la Teoría de la Autodeterminación, se predi- ce que los climas de aprendizaje que apoyan a la autonomía de los estudiantes mejorarán su motivación intrínseca (Black \& Deci, 2000; Lim \& Wang, 2009; Reeve, Jang, Carrell, Jeon, \& Barsh, 2004), mientras que los climas en los que los estudiantes perciben un control de su comportamiento, disminuye su sentido de la voluntad y se reduce la motivación natural. Aesto hay que sumarle que, según Matos (2009), la motivación intrínseca es el mejor ejemplo del comportamiento autónomo y autodeterminado. Por tanto, se puede concretar que es importante promover el apoyo a la autonomía porque esta predice la autodeterminación; y en diversas investigaciones se ha demostrado que está vinculada con una mayor concentración en la clase (Black \& Deci, 2000; Standage, Duda, \& Ntoumanis, 2005), un mayor esfuerzo académico (Ntoumanis, 2001) y un menor abandono escolar (Black \& Deci, 2000); entre otros beneficios. Por todo ello, si se pretende formar a estudiantes autónomos y con capacidad de decisión e iniciativa, el docente juega un papel fundamental pues, como demostraron Hagger, Chatzisarantis, Barkoukis, Wang y Baranowski (2005), la percepción de apoyo a la autonomía que tiene el alumno por parte del profesor en el contexto de la EF, influye en el comportamiento posterior que el estudiante tiene en su tiempo libre.

Sobre la competencia, se comprueba que existe una mejoría mayor que con la autonomía con estos contenidos, apoyando la teoría de Bisson (1999), quien manifiesta que algunos de los efectos positivos de estas actividades son el crecimiento personal y la mejora de su competencia, permitiéndoles superar los límites auto-impuestos mediante el desarrollo de habilidades para resolver los problemas planteados. Igualmente, estos datos se sitúan en la línea de Sibthorp (2003), quien manifiesta que los alumnos tras recibir un programa de EA, suelen mejorar esta variable.

La relación con los demás ha sido las variables quemás ha mejorado. Desde aquí, el docente puede diseñar tareas de aventura que ayuden a trabajar en equipo, colaborar y establecer relaciones sociales constructivas. Sobre esto, Hodge, Allen, y Smellie (2008) y Allen (2003) con-cluyeron que las personas que daban mayor importancia a las relaciones sociales en la práctica física, mostraban también mayor motivación intrínse-ca. En este trabajo, se puede apreciar tras el programa de EA, que las relaciones sociales mejoran de manera muy importante, y sobre todo, que éstas predicen la motivación intrínseca.

Por tanto, tras recibir el programa de EA, se ha comprobado que han mejorado las tres necesidades psicológicas básicas incluidas en la Teoría de las Necesidades Básicas (Deci \& Ryan (2000) y, por este motivo, es comprensible hallar como resultado la mejora de la motivación intrínseca. Esta mejora es un aspecto muy destacable de esta investigación, ya que coincide plenamente con el trabajo de Doering y Veletsianos (2008), tras aplicar un programa de EA. A esto hay que sumarle que diferentes estudios mostraron que los alumnos con una motivación autodeterminada son los que más valoran y mayor importancia dan a las clases de EF (Gómez-López, Granero-Gallegos, BaenaExtremera, \& Abraldes, 2013; Moreno \& Llamas, 2007; Moreno, Zomeño, Marín, Ruiz, \& Cervelló, 2013). Es más, existen trabajos previos que demuestran que los alumnos suelen tener una motivación buena hacia esta asignatura (Borges, Belando, \& Moreno-Murcia, 2014; Moreno-Murcia, et al., 2013), pero con la práctica de estos contenidos, esta motivación mejora aún más. Y esto es importante, pues como se ha dicho, la motivación es un factor clave que puede influir en los resultados del rendimiento académico, pues los altos logros de aprendizaje se atribuyen, a menudo, a la alta motivación de los estudiantes y a ambientes que favorecen la motivación (Moreno-Murcia, Sicilia, Cervelló, Huéscar, \& Dumitru, 2011). Es probable, que parte de este aumento en motivación, pueda venir inducida por el interés que presentan estos contenidos (Granero-Gallegos, Baena-Extremera, \& Martínez-Molina, 2010), o bien en el aumento de la autonomía en las tareas de clase hacia estos alumnos (Baena-Extremera, Granero-Gallegos, Sánchez-Fuentes, \& Martínez-Molina, 2013), propios de estas actividades. No obstante, hay que tener presente y los profesores se deberían beneficiar de esta circunstancia, que estas actividades podrían ser muy útiles para incentivar y mejorar los momentos de baja motivación de los discentes, a lo largo 
del curso y de la etapa educativa.

Otras de las variables que experimenta mejora es la satisfacción/ diversión, en contra del aburrimiento. Estos mismos resultados, ya fueron encontrados en el trabajo llevado a cabo por Baena-Extremera y Granero-Gallegos (2013) con alumnos de secundaria, tras aplicar un programa de EA. Igualmente, Ebbeck y Gibbons (1998) comprobaron cómo los estudiantes se sintieron más felices con ellos mismos, más satisfechos y aburriéndose menos. Por tanto, se puede entender como una tendencia general en estos programas, aspecto que podría ser bien aprovechado por los docentes.

En el análisis de predicción, se observa una mejora en la predicción de las tres necesidades básicas hacia la motivación intrínseca. Siguiendo el modelo jerárquico propuesto de motivación intrínseca y extrínseca por Vallerand (2001) (HMIEM), existen diversos factores sociales que influyen en la satisfacción de las necesidades psicológicas básicas de autonomía y relación con los demás para desarrollar la motivación. Así, cuando un alumno en sus clases de EF participa tomando decisiones, tienes posibilidades de elegiry además percibe una relación positiva con sus compañeros, tal como ocurre en los programas de EA, alcanzará una motivación autodeterminada, es decir, intrínseca. Y esto mismo, se refleja en los resultados de esta investigación. Esta predicción es muy importante, pues conocer como conseguir que el alumno llegue a una situación de autodeterminación favorable, supone según diversos autores, consecuencias conductuales, afectivas y cognitivas más positivas en los alumnos (González-Cutre, Sicilia, \& Fernández, 2010; Vallerand, 2007).

Recientemente existen investigaciones que han comprobado la relación predictiva entre la motivación intrínseca y la diversión en las clases deEF, como explicación causal de un modelo de satisfacción con la vida (Moreno y Vera, 2011). En los resultados encontrados en esta investigación, se apoya esta teoría, pues tal como se puede apreciar, con el trabajo de intervención se encuentran mejoras en la satisfacción/diversión y en la predicción de la motivación intrínseca hacia ésta. Estos resultados son de gran interés no solo para el docente, sino para la comunidad educativa en general pues desde la satisfacción del alumno hacia esta asignatura, podrán inculcarse hábitos de ejercicio físico e, incluso, se podrá mejorar el rendimiento escolar como ya demuestran algunos estudios (Ardoy, et al, 2013). Es más, Gehris, Kress, y Swalm (2010) comprobaron que muchos de los alumnos, tras recibir un programa de EA habían decidido, por ellos mismos, practicar actividades de aventura en su tiempo libre (como escalada) debido, principalmente, a que se habían divertido mucho con estas actividades.

Por tanto, y para concluir, la utilización de AMN, ayuda a mejorar las tres necesidades psicológicas básicas, la motivación intrínseca y la satisfacción/diversión, dándose una predicción positiva en este sentido. Esta aportación es valiosísima, pues como se sabe, los alumnos satisfechos con sus clases tienen menor estrés (Hui \& Sun, 2010), mejores relaciones sociales (Danielsen, Samdal, Hetland, \& Wold, 2009) y, por supuesto, mejor rendimiento académico (Danielsen, et al., 2009), determinando el compromiso del estudiante con su trabajo escolar(Danielsen, Breivik, \& Wold, 2011). Con este trabajo, encontramos el modo de llegar a la satisfacción/diversión, habiendo comprobado los efectos educativos de las AFM. Finalmente, destacar que el profesor de EF debería buscar actividades de EA para poder aplicarlas en el momento oportuno según la situación y necesidad actual de sus alumnos.

\section{Referencias}

Allen, J. B. (2003). Social motivation in youth sport. Journal of Sport \& Exercise Psychology, 25(4), 551-567.

Ardoy, D. N., Fernández-Rodríguez, J. M., Chillón, P., Artero, E. G. España-Romero, V., Jiménez-Pavón, D., ... Ortega, F. B. (2010). Educando para mejorar el estado de forma física, estudio EDUFIT: antecedentes, diseño, metodología y análisis del abandono/adhesión al estudio. Revista Española de Salud Publica, España, 84(2), 151168.

Baena-Extremera, A. (2011). Programas didácticos para Educación Física a través de la Educación de Aventura. Espiral. Cuadernos del
Profesorado, 4(7), 3-13. Recuperado de http://www.cepcuevasolula.es/ espiral/articulos/ESPIRAL_VOL_4_N_7_ART_1.pdf

Baena-Extremera, A. \& Granero-Gallegos, A. (2013). Efecto de un programa de Educación de Aventura en la orientación al aprendizaje, satisfacción y autoconcepto en secundaria. Revista Iberoamericana de Diagnóstico y Evaluación Psicológica, 36(1), 59-78.

Baena-Extremera, A., Granero-Gallegos, A., \& Ortiz-Camacho, M. M. (2012). Quasi-experimental study of the effect of an Adventure Education programme on classroom satisfaction, physical selfconcept and social goals in Physical Education. Psychologica Belgica, 52(4), 386-396.

Baena-Extremera, A., Granero-Gallegos, A., Pérez-Quero, F. J., \& BrachoAmador, C. (2012). Versión española del Sport Satisfaction Instrument (SSI) adaptado a la Educación Física. Revista de Psicodidáctica, 17(2), 377-395. Recuperado de http://www.ehu.eus/ojs/index.php/ psicodidactica/article/view/4037/5959

Baena-Extremera, A., Granero-Gallegos, A., Sánchez-Fuentes, J. A., \& Martínez-Molina, M. (2013). Apoyo a la autonomía en Educación Física: antecedentes, diseño, metodología y análisis de la relación con la motivación en estudiantes adolescentes. Retos, 24, 46-49. Recuperado de http://retos.org/numero_24/46-49.pdf

Balaguer, I., Atienza, F. L., Castillo, I., Moreno, Y., \& Duda, J. L. (1997). Factorial structure of measures of satisfaction/interest in sport and classroom in the case of Spanish adolescents. Abstracts of 4th. European Conference of Psychological Assessment (pp. 76). Lisbon: Portugal.

Bisson, C. (1999). Sequencing the adventure experience. En J. Miles \& S Priest (Eds.) Adventure programming (pp. 205-214). Venture: State College, PA.

Black, A. E. \& Deci, E. L. (2000). The effects of instructors' autonomy support and students' autonomous motivation on learning organic chemistry: A self-determination theory perspective. Science Education, 84, 740-756.

Borges, F., Belando, N., \& Moreno-Murcia, J. A. (2014). Perception of Equal Treatment and the Importance of Physical Education of Adolescent Girls. Revista de Psicodidáctica, 19(1), 173-189. Recuperado de http://www.ehu.eus/ojs/index.php/psicodidactica/article/ view/8007/9959

Brière, N., Vallerand, R., Blais, N., \& Pelletier, L. (1995). Développement et validation d'une mesure de motivation intrinsèque, extrinsèque et d'amotivation en contexte sportif :l‘Échelle de motivation dans les sports (ÉMS). International Journal of Sport Psychology, 26, 465489 .

Caballero, P. (2012). Potencial educativo de las actividades físicas en el medio natural: actividades de colaboración simple. EmasF, Revista Digital de Educación Física, 19,(4), 99-114. Recuperado de http:// emasf2.webcindario.com/NUMERO 19 EMASF.pdf

Danielsen, A. G., Breivik, K., \& Wold, B. (2011). Do Perceived Academic Competence and School Satisfaction Mediate the Relationships Between Perceived Support Provided by Teachers and Classmates, and Academic Initiative? Scandinavian Journal of Educational Research, 55(4), 379-401.

Danielsen, A. G., Samdal, O., Hetland, J., \& Wold, B. (2009). Schoolrelated social support and students' perceived life satisfaction. Journal of Education Research, 102(4), 303-318.

Deci, E. L. \& Ryan, R. M. (1985). Intrinsic motivation and selfdetermination in human behavior. New York: Plenum.

Deci, E. L. \& Ryan, R. M. (2000). The "what» and "why» of goal pursuits: Human needs and the self-determination of behavior. Psychological Inquiry, 11, 227-268.

Duda, J. L. \& Nicholls, J. G. (1992). Dimensions of achievement motivation in schoolwork and sport. Journal of Educational Psychology, 84(3), 290-299.

Ebbeck, V. \& Gibbons, S. (1998). The Effect of Team Building Program on the Self-Conceptions of Grade 6 and 7 Physical Education Students. Journal of Sport and Exercise Psychology, 20, 300-310.

Flintoff, A. \& Scraton, S. (2001). Stepping into Active Leisure? Young Women's Perceptions of Active Lifestyles and their Experiences of School Physical Education. Sport, Education and Society, 6(1), 521

Gatzemann, T., Schweizer, K., \& Hummel, A. (2008). Effectiveness of sports activities withs an orientation on experiential education, adventure-based learning and outdoor-education. Kinesiology, 40(2), 146-152.

Gehris, J., Kress, J., \& Swalm, R. (2010). Students' Views on Physical Development and Physical Self-Concept in Adventure-Physical 
Education. Journal of Teaching in Physical Education, 29, 146-166.

Gómez-López, M., Granero-Gallegos, A., Baena-Extremera, A., \& Abraldes, J.A (2013).Análisis de los perfiles motivacionales y su relación con la importancia de la educación física en secundaria. Revista Iberoamericana de Diagnóstico y Evaluación Psicológica,36(en prensa).

González-Cutre, D., Sicilia,A., \& Fernández,A. (2010). Hacia una mayor comprensión de la motivación en el ejercicio físico: medición de la regulación integrada en el contexto español. Psicothema, 22(4), 841-847.

Granero-Gallegos, A. \& Baena-Extremera, A. (2007). Importancia de los valores educativos de las actividades físicas en la naturaleza. Habilidad Motriz, 29, 514.

Granero-Gallegos,A. \& Baena-Extremera,A. (2013).Análisis preliminarexploratorio del «Sport Motivation Scale(SMS)» adaptado a la Educación Física. Espiral. Cuadernos del Profesorado, 6(12), 3-4. Recuperado de http:// w w w. c e p c u e va solula.e s/e s piral/articulos/ ESPIRAL VOL 6 N 12 ART 1.pdf.

Granero-Gallegos, A., Baena-Extremera,A., \& Martínez-Molina, M. (2010). Contenidos desarrollados mediante las actividades en el medio natural de las clases de Educación Física en Secundaria Obligatoria.Ágora, para la Educación Física yel Deporte, 12(3), 273-278.

Granero-Gallegos,A., Baena-Extremera,A., Gómez-López, M., Sánchez-Fuentes, J. A., \& Abraldes, J.A. (2014). Psychometric properties of the «Sport Motivation Scale(SMS)» adapted to Physical Education. Journal of Sports Science and Medicine, 13(4), 801-807. Recuperado de http://www.jssm.org/ research.php?id=jssm-13-801.xml

Hagger, M. S., Chatzisarantis, N., Barkoukis, V., Wang, C. K. J., \& Baranowski, J. (2005). Perceived autonomy support in physical education and leisure-time physical activity: a cross-cultural evaluation of the trans-contextual model. Journal of Educational Psychology, 97, 376-390.

Hair, J. F., Anderson, R. E., Tatham, R. L., \& Black, W. C. (1999). Multivariate Data Analysis. Upper Saddle River, NJ: Prentice-Hall.

Hattie, J., Marsh, H. W., Neill, J. T., \& Richards, G. E. (1997). Adventure education and Outward Bound: Out-of-class experiences that make a lasting difference. Review of Educational Research, 67, 43-87.

Hodge, K., Allen, J. B., \& Smellie, L. (2008). Motivation in Masters Sport: Achievement and social goals. Psychology of Sport and Exer-cise, 9(2), 157176.

Hooper, D., Coughlan, J., \& Mullen, M. (2008). Structural Equation Modelling: Guidelines for Determining Model Fit. Electronic Journal of Business Research Methods, 6(1), 53-60.

How, Y. M., Whipp, P. R., Dimmock, J.A., \& Jackson, B. (2013). The Effects of Choice on Autonomous Motivation, PerceivedAutonomy Support, and Physical Activity Levels in High School Physical Education. Journal of Teaching in Physical Education, 32, 131-148.

Hu, L. \& Bentler, P. M. (1999). Cutoff criteria for fit indexes in covariance structure analysis: Conventional criteria versus new alternatives. Structural Equation Modelling, 6, 1-55.

Hui, E. K. P. \& Sun, R. C. F. (2010). Chinese children's perceived school satisfaction: the role of contextual and intrapersonal factors, Educational Psychology:An Intemational. Journal of Experimental Educational Psychology, 30(2), 155-172.

Jöreskog, K. G. \& Sörbom, D. (1993). Structural equation modeling with the SIMPLIS command language. Chicago: Scientific Software International.

Kline, R. B. (2005), Principles and Practice of Structural Equation Modeling (2nd ed.). New York: The Guilford Press.

Levy, R. \& Hancock, G. R. (2007). A framework of statistical tests for comparing mean and covariance structure models. Multivariate Behavioral Research, 42, 33-66.

Lim, B. S. C. \& Wang, C. K. J. (2009). Perceived autonomy support, behavioural regulations in physical education and physical activity intention. Psychology of Sport and Exercise, 10, 52-60.

MacPhail, A., Kirk, D., \& Eley, D. (2003). Listening to young people’s voices: Youth sports leaders' advice on facilitating participation in sport. European Physical Education Review, 9, 57-73.

Markland, D. (2007). The golden rule is that there are no golden rules: Acommentary on Paul Barrett's recommendations for reporting model fit in structural equation modelling. Personality and Individual Differences, 42(5), 851-858.

Matos, L. (2009). Adaptación de dos cuestionarios demotivación:Autorregulación del Aprendizaje y Clima de Aprendizaje. Revista Persona, 12, 167-185.

Moos, D. C. \& Honkomp, B. (2011). Adventure Learning: Motivating Students in a Minnesota Middle School. Journal of Research on Technology in Education, 43(3), 231-252.

Moreno Murcia, J. A., Sicilia, A., Cervelló, E., Huéscar, E., \& Dumitru, D. C (2011). The relationship between goal orientations, motivational climate and self-reported discipline in physical education. Journal of Sport Science and Medicine, 10, 119-129. Recuperado de http://www.jssm.org/vol10/n1/16/ v10n1-16text.php
Moreno-Murcia, J. M., Zomeño, T., Marín de Oliveira, L. M., Ruiz, L. M., \& Cervelló, E. (2013). Percepción de la utilidad e importancia de la educación física según la motivación generada por el docente. Revista de Educación, 362, 380-401. Recuperado de http://www.mecd.gob.es/dctm/revista-de-educacion/ articulosre362/re36214.pdf?documentId=0901e72b816fbabc

Moreno, J. A. \& Vera, J. A. (2011). Modelo causal de la satisfacción con la vida en adolescentes de educación física. Revista de Psicodidáctica, 16(2), 367-380. Recuperado dehttp://www.ehu.eus/ojs/index.php/psicodidactica/article/view/ 806

Moreno, J.A., \& Llamas, L. S. (2007). Predicción de la importancia concedida a la EF según el clima motivacional y la motivación autodeterminada en estudiantes adolescentes. Enseñanza, 25, 137-155.

Moreno, J. A., González-Cutre, D., Chillón, M., \& Parra, N. (2008). Adaptación a la educación física de la escala de las necesidades psicológicas básicas en el ejercicio. Revista Mexicana de Psicología, 25(2), 295-303.

Moreno, J. A., Llamas, L. S., \& Ruiz, L. M. (2006). Perfiles motivacionales y su relación con la importancia concedida a la Educación Física. Psicología Educativa, 12(1), 49-63.

Moreno, J. A., Parra, N., \& González-Cutre, D. (2008). Influencia del apoyo a la autonomía, las metas sociales y la relación con los demás sobre la desmotivación en educación física. Psicothema, 20(4), 636-641.

Mowling, C. M., Brock, S. J., Eiler, K. K., \& Rudisill, M. E. (2004). Student motivation in physical education: breaking down barriers, Journal of Physical Education, Recreation and Dance, 75, 40-51.

Ntoumanis, N.(2001).Aself-determination approach to the understanding of motivation in physical domain. British Journal of Educational Psychology, 71, 225-242.

Ntoumanis, N. (2005). A prospective study of participation in optional school physical education using a self-determination theory framework. Journal of Educational Psychology, 97, 444-453.

Nunnally, J. C. \& Bernstein, I. J. (1995). Teoría psicométrica. Madrid: McgrawHill.

Pelletier, L. G., Fortier, M. S., Vallerand, R. J., Tuson, K. M., Brière, N. M., \& Blais, M. R. (1995). Toward a new measure of intrinsic motivation, extrinsic motivation, and amotivation in sports: the Sport Motivation Scale (SMS). Journal of Sport and Exercise Psychology, 17, 35-53.

Reeve, J., Jang, H., Carrell, D., Jeon, S., \& Barsh, J. (2004) 'Enhancing students' engagement by increasing teachers' autonomy support'. Motivation and Emotion, 28, 147-169.

Rhonke, K. (1989). Cows tails and Cobras II. Dubuque, IA: Kendall/Hunt.

Rikard, G. L \& Banville, D. (2006). High school student attitudes about physical education. Sport, Education and Society, 11(4), 385-400.

Sibthorp, J. (2003). An empirical look at Walsh and Golins' adventure education process model: Relationships between antecedent factors, perceptions of characteristics of an adventure education experience, and changes in self-efficacy. Journal of Leisure Research, 35, 80-106.

Standage, M. \& Treasu, D. C. (2002). Relationship among achieve-ment goal orientations and multidimensional situational motivation in physical education. British Journal of Educational Psychology, 72(1), 87-103.

Standage, M., Duda, J. L., \& Ntoumanis, N. (2005). A test of self-determination theory in school physical education. British Journal of Educational Psychology, 75, 411-433.

Steiger, J. (2007). Understanding the limitations of global fit assessment in structural equation modelling. Personality and Individual Differences, 42(5), 893-898.

Vallerand, R. J. (2001). Ahierarchical model of intrinsic and extrinsic motivation in sport and exercise. En G C. Roberts (Ed.), Advances in motivation in sport and exercise (pp. 263-319). Champaign, IL: Human Kinetics.

Vallerand, R. J. (2007). Intrinsic and Extrinsic Motivation in Sport and Physical Activity. En G Tenenbaum \& R. C. Eklund (Eds.), Handbook of sport Psychology ( $3^{\mathrm{a}}$ ed., pp. 59-83). New York: Wiley.

Vlachopoulos, S. P. \& Michailidou, S. (2006). Development and initial validation of a measure of autonomy, competence, and relatedness in exercise: The Basis Psychological Needs in Exercise Scale. Measurement in Physical Education and Exercise Science, 10, 179-201.

Wurdinger, S. \& Steffen, J. (2003). Developing challenge course programs for schools. Dubuque, IA: Kendall Hunt.

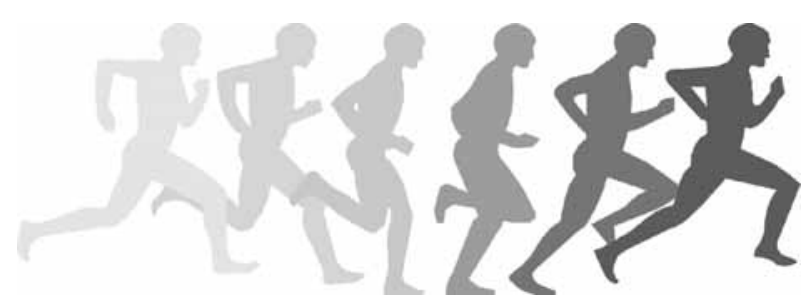

